\title{
GESTIÓN DE EDUCACIÓN VIRTUAL
}

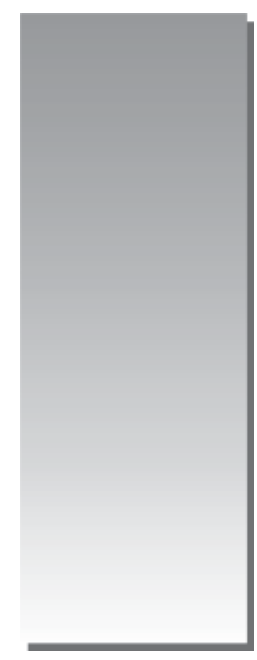

Juan Castillo Maza*

E-mail: jcastillom@unmsm.edu.pe

\section{RESUMEN}

La apertura social, además de la apertura espacio-tiempo, hacen posible que una institución educativa o un establecimiento asociado atiendan a un número significativamente grande de personas que aún encontrándose dispersas geográficamente y lejos de sus docentes, satisfacen necesidades de diversa índole como formación, capacitación, actualización, orientación, especialización y perfeccionamiento, según sea el caso.

La educación virtual es una modalidad de la educación a distancia y abierta que en un contexto de nuevos ambientes telemáticos posibilita la comunicación humana mediada por la tecnología de información y comunicaciones, que acortan la distancia entre la enseñanza y el aprendizaje. En esta nueva forma de interacción global, profesores y estudiantes pueden compartir todo tipo de mensajes educativos en tiempo real o en forma asincrónica. El espacio donde se desarrolla la educación virtual es el ciberespacio generado por Internet.

La gestión de educación virtual garantizará una buena ejecución de los planes y programas educativos en la educación a distancia y abierta en la modalidad virtual.

Palabras clave: Gestión, e-learning.

\section{ABSTRACT}

The social opening besides of the opening space - time, makes possible that an educational institution or an establishment associated to this, assist to a significantly big number of people that even being dispersed geographically and far from its teachers satisfy necessities of diverse nature, as it is the case: formation, qualification, upgrade, orientation, specialization and improvement.

The virtual education is a modality of the education to distance and open that make possible in a context of telematic new atmospheres the human communication mediated by the technology of information and communications that shorten the distance between the teaching and the learning. In this new form of global interaction, professors and students can share all type of educational messages in realtime or in asynchronous form. The space where the virtual education is developed is the cyberspace generated by Internet.

* Magíster en Economía con mención en Métodos Cuantitativos y Doctor en Ciencias Contables y Empresariales. Licenciado en Administración. Profesor Principal de la UNMSM; Profesor de Post Grado en la UNMSM, UNFV, UNASAM, UNSCH, UNEGV, UNJBG, UNSAAC, UNP, UNCP y UNA. 
The management of virtual education will guarantee a good execution of the plans and educational programs in the education at distance and opened up in the virtual modality.

Keywords: Management, e-learning.

\section{INTRODUCCIÓN}

Los avances en el campo de la informática y las telecomunicaciones, así como el auge del conocimiento generado por estas tecnologías, son factores decisivos en la búsqueda de soluciones alternativas, tanto para la economía como para la sociedad en general ${ }^{1}$.

Las nuevas tecnologías de información y comunicaciones (NTICs) han incorporado nuevos retos y posibilidades en el ámbito de la educación. Conscientes de este nuevo entorno, presentamos el presente estudio que busca sensibilizar y luego implementar el servicio de educación a distancia en la modalidad virtual en la Facultad de Ciencias Administrativas de la Universidad Nacional Mayor de San Marcos, en los niveles de post grado (Diplomados, Maestrías y Doctorados), así como cursos de especialización orientados a los trabajadores de empresas e instituciones publicas y privadas, locales, regionales, nacionales e internacionales.

La realización del presente estudio se ha nutrido con la visita a instituciones educativas con una larga experiencia en procesos de enseñanza a distancia en la modalidad virtual como la Universidad de Guadalajara en México, que tiene una organización propia para el sistema virtual como unidad académica autónoma dentro de la estructura general. Adicionalmente a ello hemos participado en diversos eventos especializados en la virtualización de la educación como el XIV Encuentro Internacional de Educación a Distancia: Gestar y Gestionar la Virtualidad. Realidades y Virtualidades en la Educación (Guadalajara, México); Nova Educa 2006 (Miami, Estados Unidos); el IV Congreso Internacional de Educación a Distancia: Universidad 2006 Universalización de la Universidad (La Habana, Cuba); el VII Encuentro Internacional Virtual Educa sobre Educación, Innovacion y Cooperación (Bilbao, España) y el Primer Congreso Internacional de Educación a Distancia (Lima, Perú).

\section{MODELOS DE EDUCACIÓN VIRTUAL}

En el contexto internacional vamos a referirnos a dos instituciones que desarrollan con éxito el modelo de educación a distancia en la modalidad virtual. Ellas son la Universidad Nacional de Educación a Distancia (UNED) ${ }^{2}$ de España y el Sistema de Universidad Virtual (UDG VIRTUAL) ${ }^{3}$ de la Universidad de Guadalajara Jalisco, México.

\section{UNIVERSIDAD NACIONAL DE EDUCACIÓN A DISTANCIA (UNED) DE ESPAÑA}

El modelo educativo de la UNED está basado en la creencia de que la relación directa profesor/alumno no es completamente imprescindible, ya que es posible aprender en solitario determinados contenidos científicos, siempre que se use una metodología adecuada que garantice la calidad y claridad de los mismos, y se disponga de los cauces tecnológicos adecuados para asegurar una comunicación fluida con el profesor. Para lograr este objetivo se necesitan instrumentos pedagógicos de una calidad universitaria contrastada que se materializan en el material didáctico.

El modelo de la UNED tiene tres características: en primer lugar, la metodología a distancia y el uso intensivo de las nuevas tecnologías de la información y la comunicación, desde Internet hasta la televisión educativa o los programas de radio. En segundo lugar, la UNED cuenta con una extensa red de Centros Asociados dentro y fuera de España que permiten al estudiante acercarse a un centro universitario, consultar con su profesor-tutor, realizar sus exámenes, y acceder a servicios informáticos y bibliotecarios de excelente nivel. En tercer lugar, la UNED presta especial interés a la dimensión investigadora propia de toda universidad que se precie, y que se plasma tanto en la innovación en diversos campos de conocimiento, cuanto en el desarrollo de metodologías de enseñanza que permitan aprovechar los resultados obtenidos. En este sentido, la investigación llevada a cabo por grupos de excelencia y equipos de investigación sitúa a la UNED en una posición relevante dentro del conjunto de las universidades españolas.

La Universidad Nacional de Educación a Distancia (UNED) es una universidad de ámbito nacional, que oferta en la actualidad veinte li- 
cenciaturas, cuatro diplomaturas, tres ingenierías y siete ingenierías técnicas. Tras más de treinta años de funcionamiento, es una universidad que goza de un prestigio merecido, siendo la universidad con más alumnos matriculados de toda España. Con una voluntad de mejora constante, equipos de investigación líderes en su campo, y una metodología de enseñanza a distancia contrastada, la UNED se ha consolidado en estos años como un referente dentro del panorama universitario español.

La UNED, para el cumplimiento de su misión y visión, cuenta con los siguientes Órganos de Gobierno: Órganos Colegiados, Órganos Unipersonales y Órganos de Facultades y de Escuelas Técnicas Superiores. Entre los Órganos Colegiados figuran el Claustro Universitario, el Consejo de Gobierno y el Consejo Social. Los Órganos Unipersonales se constituyen por el Rector, Vicerrectores, Secretario General y el Gerente. Entre los Órganos de Facultades y de Escuelas Técnicas figuran las Juntas de Facultades y Escuelas Técnicas Superiores, los Consejos de Departamento, los Decanos de Facultad / Directores de ETS. y Directores de Departamento.

\section{El Claustro Universitario}

Según lo establecido en el Artículo № 16 de la Ley Orgánica de Universidades, es el máximo órgano de representación de la comunidad universitaria. De conformidad con lo establecido en el Artículo № 78 de los Estatutos de la Universidad Nacional de Educación a Distancia (UNED), está compuesto por el Rector, que lo presidirá, la Secretaría General, el Gerente y por 297 representantes elegidos por los distintos sectores de la comunidad universitaria.

Son funciones del Claustro:

a) Elaborar y modificar los Estatutos.

b) Elaborar, aprobar y modificar su propio reglamento.

c) Aprobar el reglamento de Funcionamiento del Defensor Universitario, así como elegirlo y debatir su informe anual.

d) Convocar a elecciones extraordinarias para elegir rector en los términos fijados en la Ley Orgánica 6/2001 del 21 de diciembre, y en estos estatutos.

e) Elegir a los representantes de los distintos sectores del claustro en el Consejo de Gobierno. f) Deliberar sobre las líneas generales de actuación de la universidad en materia de docencia e investigación prevista por el Consejo de Gobierno.

g) Formular recomendaciones, propuestas y declaraciones institucionales, así como debatir los informes que le sean presentados por el Rector, el Consejo de Gobierno u otros órganos o miembros de la comunidad universitaria.

h) Recabar cuanta información estimen necesaria acerca del funcionamiento de la universidad y solicitar la comparecencia de los representantes de cualquier órgano o servicio universitario.

i) Designar, a propuesta del Consejo de Gobierno, los siete catedráticos que han de formar parte de la Comisión de Reclamaciones a que se refiere el Artículo № 66 de la Ley Orgánica 6/2001 del 21 de diciembre.

j) Cualquier otra que le sea atribuida por estos estatutos y las restantes normas de aplicación.

\section{El Consejo de Gobierno}

Es el órgano de gobierno de la Universidad. Establece las líneas estratégicas y programáticas de la Universidad así como las directrices y procedimientos para su aplicación, en los ámbitos de organización de la enseñanza, investigación, recursos humanos y económicos y elaboración de los presupuestos. Ejerce las funciones previstas en la Ley Orgánica de Universidades (LOU) y las que establezcan los Estatutos.

De conformidad con el Artículo №82 de los Estatutos de la UNED, el Consejo de Gobierno quedará compuesto de la siguiente forma:

a) Miembros natos: Rector, la Secretaria General y el Gerente.

b) Quince miembros designados por el Rector.

c) Veinte miembros elegidos por el Claustro entre sus representantes.

d) Quince miembros en representación de los Decanos de Facultad, Directores de Escuela, Directores de Departamento y Directores de Instituto.

e) Tres miembros en representación del Consejo Social. 
f) Un representante de personal de Administración y Servicios de Centros Asociados (con voz y sin voto).

\section{El Consejo Social}

Es el órgano de participación de la sociedad en la Universidad. Corresponde al Consejo Social la supervisión de las actividades de carácter económico de la Universidad y del rendimiento de sus servicios; promover la colaboración de la sociedad en la financiación de la Universidad, y las relaciones entre ésta y su entorno cultural, profesional, económico y social al servicio de la calidad de la actividad universitaria, a cuyo fin podrá disponer de la oportuna información de la Agencia Nacional de Evaluación de la Calidad y Acreditación.

Asimismo, le corresponde la aprobación del presupuesto y de la programación plurianual de la Universidad, a propuesta del Consejo de Gobierno. Además, con carácter previo al trámite de rendición de cuentas a que se refieren los Artículos № 81 y № 84 de la LOU, le corresponde aprobar las cuentas anuales de la Universidad y las de las entidades que de ella puedan depender y sin perjuicio de la legislación mercantil u otra a las que dichas entidades puedan estar sometidas en función de su personalidad jurídica.

La Ley regulará la composición y funciones del Consejo Social y la designación de sus miembros de entre personalidades de la vida cultural, profesional, económica, laboral y social, que no podrán ser miembros de la propia comunidad universitaria. Serán, no obstante, miembros del Consejo Social, el Rector, el Secretario general y el Gerente, así como un profesor, un estudiante y un representante del personal de administración y servicios, elegidos por el Consejo de Gobierno de entre sus miembros. El Presidente del Consejo Social de la UNED será nombrado por el Gobierno.

\section{El Rector}

Según el Artículo № 20 de la Ley Orgánica de Universidades el Rector, máxima autoridad académica de la Universidad, es elegido por la Comunidad universitaria, en votación nominal y secreta, de entre los Catedráticos que presten sus servicios en ella. Son funciones del Rector: a) Representar a la Universidad.

b) Nombrar y cesar libremente a los Vicerrectores y Secretario General.

c) Nombrar y cesar al Gerente de acuerdo con el Consejo Social.

d) Ejecutar los acuerdos del Claustro Universitario, del Consejo de Gobierno y del Consejo Social.

e) Redactar la Memoria Anual de su gestión y presentarla al Claustro.

f) Aquellas expresamente delegadas por los órganos colegiados de gobierno.

g) Cualesquiera otras que no hayan sido expresamente atribuidas a otros órganos de la Universidad.

Los Vicerrectores, según el Artículo № 21 de la Ley Orgánica de Universidades, son nombrados de entre los Profesores Doctores que presten sus servicios en la Universidad.

El Secretario General, según lo dispuesto en la Ley Orgánica de Universidades, es nombrado por el Rector de entre los Funcionarios Públicos del Grupo A que presten servicios en la Universidad. Además de las funciones que le son atribuidas por la Ley o por otros preceptos de los Estatutos, corresponde al Secretario General la condición de fedatario de la documentación oficial de la Universidad.

El Gerente, según lo dispuesto en la Ley Orgánica de Universidades, tendrá que ser titulado superior y es nombrado por el Rector de acuerdo con el Consejo Social. Corresponde al Gerente la gestión de los servicios administrativos y económicos de la Universidad, y no podrá ejercer funciones docentes.

Las Juntas de Facultades y Escuelas Técnicas Superiores, según el Artículo № 18 de la Ley Orgánica de Universidades, están presididas por el Decano o Director y son el órgano de gobierno de las Facultades y Escuelas Técnicas.

Los Consejos de Departamento, presididos por su Director, son los órganos de gobierno de los mismos. Están integrados por los Doctores miembros del Departamento, así como por una representación del resto del personal docente e investigador no Doctor, en la forma que determinen los Estatutos. 
Los Decanos de Facultad y/o Directores de las Escuelas Técnicas Superiores (ETS), ostentan la representación de sus centros y ejercen las funciones de dirección y gestión ordinaria de los mismos. Serán elegidos, en los términos establecidos por los Estatutos, entre profesores Doctores pertenecientes a los cuerpos docentes universitarios adscritos al respectivo centro.

Los Directores de Departamento ostentan la representación de éste y ejercen las funciones de dirección y gestión ordinaria del mismo. Serán elegidos por el Consejo de Departamentos en los términos establecidos por los Estatutos, entre profesores Doctores pertenecientes a los cuerpos docentes universitarios miembros del mismo.

\section{SISTEMA DE UNIVERSIDAD VIRTUAL (UDG VIRTUAL) DE LA UNIVERSIDAD DE GUADALA- JARA, JALISCO-MÉXICO}

La UDG Virtual se plantea como el órgano desconcentrado de la Universidad de Guadalajara responsable de administrar y desarrollar programas académicos de nivel medio-superior y superior, en modalidades no escolarizadas apoyadas en las tecnologías de la información y de la comunicación, para la comunidad universitaria y sociedad en general.

Tiene como campo de saber los procesos de gestión de conocimiento y de aprendizaje, con el aprovechamiento de entornos virtuales y por sus características fortalece a la Red Universitaria de la Universidad de Guadalajara y a la cooperación regional, nacional e internacional.

Cuenta con cuerpos académicos especializados en los procesos de aprendizaje y de gestión del conocimiento, especialmente cuando en entornos virtuales, por lo que tiene una oferta académica propia y apoya a todas las áreas del conocimiento que se desarrollen en la institución.

El Sistema de Universidad Virtual (UDG VIRTUAL) de la Universidad de Guadalajara tiene su antecedente en 1990, cuando surgió el proyecto "Sistema de Universidad Abierta y a Distancia" cuyo objetivo era diseñar un sistema de educación abierta y a distancia que abriera la posibilidad de formación certificada a una población que no podía realizar estudios formales de tipo pre- sencial. Posteriormente en 1992 se dictaminó la creación de la División de Educación Abierta y a Distancia (DEAD), dependiente de la Dirección de Desarrollo Académico, con el propósito de promover el desarrollo institucional de la Universidad y generar opciones de innovación educativa acordes con los requerimientos de la educación superior en la región. En 1994 se creó la Coordinación de Educación Continua, Abierta y a Distancia (CECAD), uno de cuyos propósitos era hacer que los requerimientos sociales de formación y actualización, considerados como un compromiso de la Universidad con la comunidad, lograran mayor pertinencia, calidad y eficiencia.

En 1999 se creó la Coordinación General del Sistema para la Innovación del Aprendizaje (INNOVA), dependencia que tiene entre sus funciones:

a) Desarrollar modelos, ambientes y contenidos de aprendizaje en red, conjuntamente con los Centros Universitarios y el Sistema de Educación Media Superior.

b) Diseñar programas educativos en modalidades no convencionales, utilizando para ello las aportaciones de la ciencia y las tecnologías de la información y la comunicación.

c) Promover la investigación para la diversificación y desarrollo de ambientes de aprendizaje y prácticas educativas innovadoras.

El 01 de enero del 2005 desaparece la Coordinación General del Sistema para la Innovación del Aprendizaje y nace el Sistema de Universidad Virtual, el cual tiene entre sus atribuciones:

a) Ofrecer programas educativos.

b) Certificar estudios.

c) Realizar investigación.

d) Realizar actividades de extensión, vinculación y difusión.

e) Proponer al Consejo General Universitario los planes de estudio que ofrecerá.

La organización del Sistema de Universidad Virtual (UDG VIRTUAL) de la Universidad de Guadalajara es dirigida por un Rector del Sistema y tres Direcciones: Dirección Académica, Dirección de Tecnologías y Dirección Administrativa (Ver Gráfico № 1). 
Gráfico № 1. Organización del Sistema de Universidad Virtual (UDG VIRTUAL). Universidad de Guadalajara.

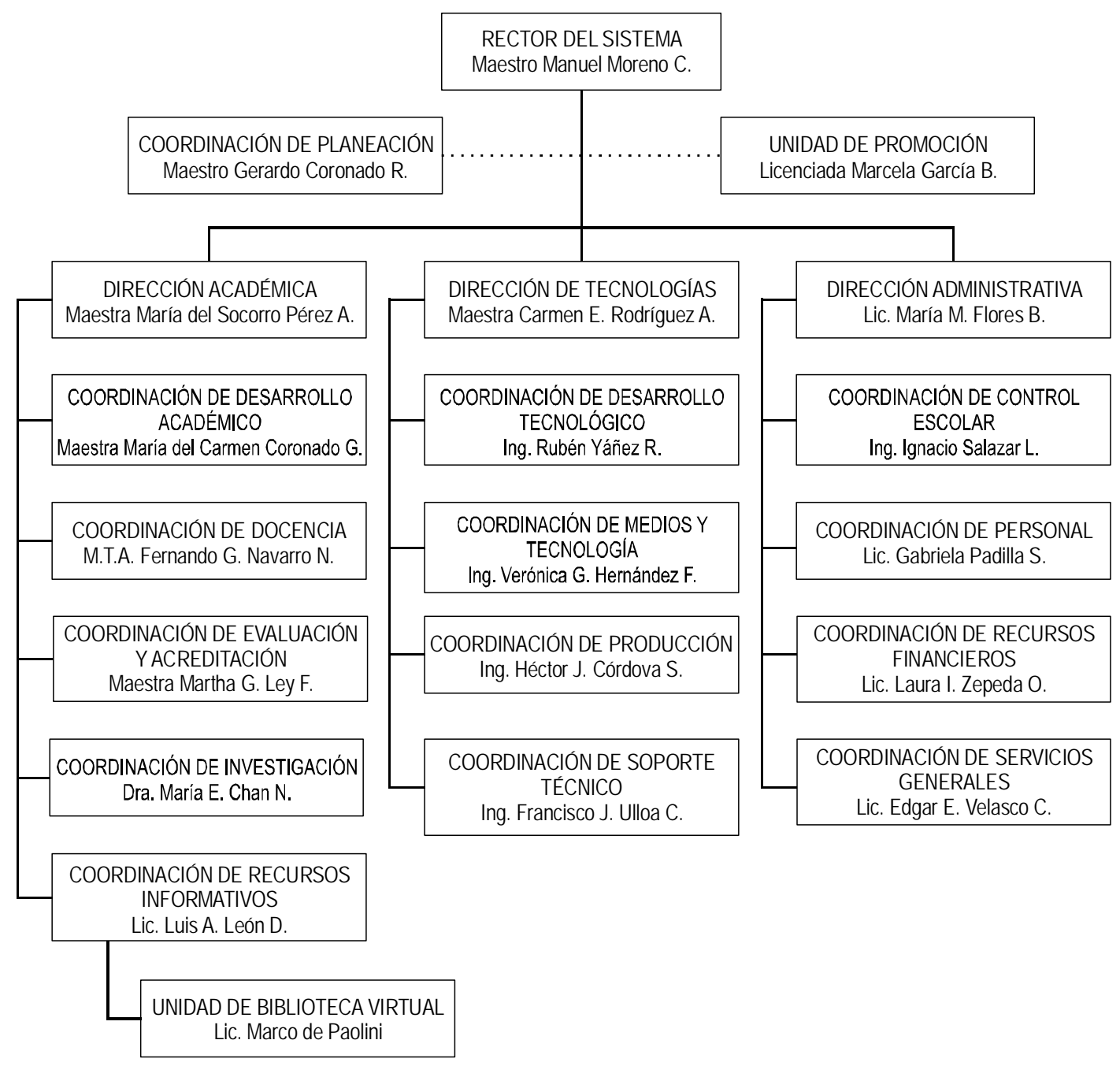

La propuesta de UDG VIRTUAL involucra un modelo pedagógico centrado en el estudiante, en el que se involucran apoyo administrativo, atención personal, soporte tecnológico y servicios académicos. El estudiante se ve inmerso en procesos y ambientes para el aprendizaje sustentados en dimensiones de aprendizaje como el perceptual, cognitivo, afectivo, social, interaprendizaje, intraprendizaje, hiperaprendizaje, metaaprendizaje, autoaprendizaje, aprendizaje significativo, aprendizaje autogestivo y aprendizaje anticipatorio.

El modelo propuesto por la UDG VIRTUAL está caracterizado por ser no convencional, es por esto que apropia las modalidades de educación continua, abierta, a distancia, en ambientes virtuales, docencia no convencional y desarrollo instruccional.
La evaluación esta diseñada para seguir, medir y guiar los aprendizajes del conjunto académico; es decir, estudiante, profesor y tutor. El modelo tecnológico esta basado en cómputo y red que permite sustentar todo un sistema complejo, diseñado para facilitar los aprendizajes en un marco de innovación y mejora continua.

\section{GESTIÓN ESTRATÉGICA DE LA EDUCACIÓN VIRTUAL}

El modelo general de la administración estratégica ${ }^{4}$-desarrollar una misión y visión estratégicas; realizar auditoría externa e interna, establecer objetivos a largo plazo; generar, evaluar y seleccionar estrategias; establecer políticas y objetivos anuales; asignar recursos así como medir y evaluar resultados- que corresponde al proceso general de formular 
estrategias, implementar estrategias, evaluar estrategias y la retroalimentación con el propósito de reciclar según se necesite mejorar y/o cambiar, resulta apropiado asumir en la gestión de educación virtual para garantizar el éxito de esta nueva modalidad de educación a distancia con resultados óptimos, es decir, beneficiar a un número cada vez más creciente de usuarios del sistema.

La gestión estratégica del modelo del campus virtual ${ }^{5}$ engloba las siguientes tareas: creación del plan estratégico del negocio y contactos iniciales para acuerdos estratégicos, como punto de partida para el desarrollo e implantación de un proyecto de tamaña envergadura, estableciendo claramente y desde una perspectiva a corto y medio plazo todos los aspectos relacionados al modelo. Los contactos iniciales para acuerdos estratégicos vía alianzas que, $a$ priori, parecen interesantes para el desarrollo exitoso de los negocios en red deben ser alianzas con empresas de tecnología, con empresas on-line, con proveedores y distribuidores y/o vendedores, y alianzas con empresas de logística.

\section{ANÁLISIS Y DISCUSIÓN}

La discusión sobre el tema de la educación a distancia en la modalidad virtual se realiza en todos los niveles y ámbitos. La educación virtual no es solo el uso de las nuevas tecnologías de información y comunicaciones (NTICs) sino que incluye el modelo de enseñanza, filosofía y plataforma tecnológica.

La calidad es otro tema que se exige a la educación virtual, pero ésta no debería exigirse solo en la modalidad virtual sino que también debe exigirse en la modalidad presencial. Es preciso indicar que aún no existen indicadores o estándares que permitan evaluar la educación a distancia en la modalidad virtual. Tanto la enseñanza presencial como el e-learning íntegramente virtual deben coexistir, de tal forma que se plantea el modelo del blended learning o b-learning es decir una parte presencial y la otra virtual.

Bajo el principio de universalización de la universidad se busca ampliar la cobertura de la educación a un mayor numero de beneficiarios; eso se puede lograr utilizando la modalidad virtual que reduce costos, amplía el número de beneficiarios, y permite hacer un mayor seguimiento al proceso de enseñanza-aprendizaje.
Para adaptarse a los cambios que se generan constantemente es necesario contar con información relevante del mercado, competencia, innovaciones tecnológicas, tendencias políticas, etc.; esto hace que la información se considere un activo empresarial, obligando a todos los miembros de una organización a tener capacidades y actitudes que les permitan encontrar y producir la información que necesitan, para luego procesarla y utilizarla de manera apropiada.

\section{CONCLUSIONES}

1. Cada día se hace más indispensable ampliar la cobertura de la educación de calidad a todos los niveles, para satisfacer necesidades educativas de formación, capacitación, actualización, orientación y perfeccionamiento.

2. La formación de recursos humanos que asumirán labores docentes en la educación virtual se hace indispensable toda vez que deben atender necesidades en nuevos entornos.

3. La flexibilidad temporal y espacial, acceso a un elevado número de participantes, disminución de costos y mayor seguimiento de la acción formativa, constituyen las principales ventajas de la educación a distancia en la modalidad virtual.

4. La organización de la educación virtual se hace tomando en cuenta tres áreas: académica, administrativa y tecnológica.

5. Ninguna universidad o centro de formación debe perder la oportunidad de incorporarse a las redes educativas que desarrollan el e-learning.

\section{NOTAS}

1. Rodríguez, M; Estrada, V; Febles, J; García, F; Castillo, J. (2005). Gestión de la educación virtual para la formación continua de profesionales de perfil empresarial. En: Gestión en el Tercer Milenio. Revista de la Facultad de Ciencias Administrativas (UNMSM). Lima, Edición 16, pp. 93-103.

2. Ver página web: www.uned.es

3. Ver página web: www.udgvirtual.udg.mx

4. Fred, R. David. (2000). Conceptos de Administración Estratégica. 5a Ed. México, Pearson Educación, p. 13.

5. Fernández, Eva I. (2004). E-Learning. Implantación de proyectos on-line. México, Alfaomega Editores, p. 35.

\section{BIBLIOGRAFÍA}

Arboleda Toro, Néstor. (2005). ABC de la educación virtual y a distancia. E-Learning en la 
Sociedad de la Información. Bogotá, Interponed - UNESCO - IESALC.

Chacón, Favio J. y Sancho, Joana. (1995). Enfoques sobre evaluación de los aprendizajes en educación a distancia I. Cuadernos de Educación a Distancia. Jalisco, Universidad de Guadalajara.

Gómez, Margarita Victoria. (2004). Educación en red. Una visión emancipadora para la formación. Jalisco, UDG Virtual de la Universidad de Guadalajara.

Fernández, Eva I. (2004). E-learning. Implantación de Proyectos de formación on-line. México D.F., Alfaomega Grupo Editor.
Fred, David. (2000). Conceptos de Administración Estratégica. México, Pearson Educación.

Poe, Richard. (2005). El Liderazgo al estilo Ola 4. Construyendo Redes. Buenos Aires, TM Editores.

Rodríguez Andino, Milagro y otros. (2005). Gestión de la educación virtual para la formación continua de profesionales de perfil empresarial. En: Gestión en el Tercer Milenio. Revista de Investigación de la Facultad de Ciencias Administrativas (UNMSM). Volumen 8, № 15, julio del 2005.

UDG Virtual de la Universidad de Guadalajara. (2005). Apertura. Revista de innovación educativa. Jalisco, Universidad de Guadalajara. 